Vol. 5, No. 02; 2021

ISSN: 2581-3366

\title{
Risk-Reducing Salpingo-Oophorectomy: A Series of 90 BRCA1 and BRCA2 Mutation Carriers and Literature Review
}

Authors:

Vanesa Rodríguez-Fernández, $\mathrm{MD}^{1,2 ;}$ Lucía Cameselle-Cortizo, $\mathrm{Md}^{1}$; Laura González Rodríguez, $\mathrm{MD}^{2}$; Elena Figueiredo-Alonso, $\mathrm{MD}^{3}$; María José Lamas González, $\mathrm{MD}^{2,4}$; Javier Valdés-Pons, $\mathrm{MD}, \mathrm{PhD}^{1,2}$; Jorge F Cameselle-Teijeiro $\mathrm{MD}, \mathrm{PhD}^{1}$.

${ }^{1}$ Clinical Oncology Research Center ADICAM. Cangas (Spain).

${ }^{2}$ Service of Obstetrics and Gynecology.álvaro Cunqueiro Hospital. Vigo (Spain).

${ }^{3}$ Service of Obstetrics and Gynecology. Povisa Hospital. Vigo (Spain)

${ }^{4}$ Breast Pathology Unit. Meixoeiro Hospital. Vigo (Spain).

Corresponding Author:

Vanesa Rodríguez Fernández, MD.

Service of Obstetrics and Gynecology. Vigo University Hospital Complex, Álvaro Cunqueiro Hospital. Estrada Clara Campoamor No 341 - 36312 Vigo. Pontevedra (Spain).

Phone: 0034986825130 - 0034986825137

doi: $10.51505 / \mathrm{ijmshr} .2021 .5212$

URL: http://dx.doi.org/10.51505/ijmshr.2021.5212

\begin{abstract}
As long as we do not have an effective screening routine, risk-reducing salpingo-oophorectomy (RRSO) is considered the gold standard for ovarian, fallopian, and primary peritoneal cancer prevention in women with documented BRCA1/2 mutations or other mutations with increased risk of ovarian cancer. Our objective was to review the pathological findings of RRSO in BRCA mutations carriers in order to estimate the prevalence of occult ovarian/tubal carcinoma. We studied a series of 90 women BRCA1/2 mutation carriers, with or without previous breast cancer, who underwent RRSO in the hospitals of our health area of Vigo. In our series, 4 women $(4.4 \%)$ were diagnosed with malignant lesions in the fallopian epithelium (all of them in early stages). The main predictor for detecting occult malignancy in women at high risk for ovarian cancer who are going to undergo RRSO is to adhere to the surgical-pathological protocol. However, we must always bear in mind that there will always be a residual risk of primary peritoneal carcinoma after RRSO (1.1\% in our series). We emphasize the importance of performing a germ line testing for all women diagnosed with epithelial ovarian/fallopian/peritoneal cancer (NCCN Guidelines). Our data support the indication for RRSO in selected high-risk patients; and recall the importance of the identification of high-risk patients, in order to offer genetic counseling and preventive measures, both for patients and their families
\end{abstract}

Keywords: BRCA genes, Breast cancer, Fallopian cancer, Ovarian cancer, Primary peritoneal cancer, Risk-reducing salpingo-oophorectomy. 
Vol. 5, No. 02; 2021

ISSN: $2581-3366$

\begin{tabular}{|c|c|}
\hline \multicolumn{2}{|c|}{\begin{tabular}{c|c} 
Acronyms \\
$R R S O$
\end{tabular}} \\
\hline \begin{tabular}{|l}
$\begin{array}{l}\text { Risk-reducing salpingo- } \\
\text { oophorectomy }\end{array}$ \\
\end{tabular} & RRSO \\
\hline \begin{tabular}{|l|} 
Breast cancer \\
\end{tabular} & $\mathrm{BC}$ \\
\hline
\end{tabular}

\section{Introduction}

As long as we do not have an effective screening routine, risk-reducing salpingo-oophorectomy (RRSO) is considered the gold standard for ovarian, fallopian, and primary peritoneal cancer prevention in women with documented BRCA1 and BRCA2 (BRCA1/2) mutations or family history consistent with hereditary breast and ovarian cancer syndrome. It is necessary to improve the identification of high-risk women who are candidates for this prophylactic surgical technique. RRSO entails a series of consequences (sterility, surgical menopause, modifications in the sexual sphere, osteoporosis...) that we have to contrast with the related benefits (avoid the morbidity and mortality of ovarian, fallopian or primary peritoneal cancer). However, we have to bear in mind that there will always be a residual risk after RRSO of being diagnosed with a primary peritoneal carcinoma. More studies are needed to gauge the risks and benefits of RRSO.

The purposes of this article were to study the pathological findings of RRSO specimens performed on women carrying a mutation in the BRCA1/2 gene and to review the literature in this regard.

\section{Material y Methods}

We studied a series of women who underwent RRSO in the hospitals of the health area of southern Pontevedra (University Hospital Complex of Vigo, University Hospital Complex of Pontevedra, and Povisa Hospital of Vigo). We present the pathological findings of RRSO performed on women with documented BRCA1/2 mutations. Age at diagnosis, mutation detected, date of RRSO, and personal oncological history before and after the prophylactic surgery was also included. As a curiosity, we also include 4 patients with breast cancer (BC) who did not undergo RRSO and were later diagnosed with high-grade serous carcinomas of the ovary (at 49, 60, and 82 years) and a serous carcinoma of the endometrium (at 46 years). The medical records were studied retrospectively and prospectively. The data obtained in this study were entered into a computer database developed in the Microsoft Excel program.

\section{Results}

We present the pathological findings of RRSO performed on 90 women with documented BRCA1/2 mutations: 52 had previously a $\mathrm{BC}$ and 38 women without this oncological history (although 5 of them were subsequently diagnosed with BC) (Table 1).

It is striking that in up to 4 women (4.4\%) malignant lesions were diagnosed at the level of the tubal epithelium. One of them was a healthy woman with a BRCA1 mutation (known since 2006) who was diagnosed ten years later with serous tubal infiltrating carcinoma (pT1a) at the time of RRSO. Another healthy woman with a BRCA1 mutation (known since 2015) underwent 
Vol. 5, No. 02; 2021

ISSN: 2581-3366

RRSO four years later, finding a serous tubal intraepithelial carcinoma. Among the group of BC patients, one of them was diagnosed with BC at age 46 (2011) and the BRCA1 mutation was identified in 2016; this patient underwent RRSO and was diagnosed with serous tubal intraepithelial lesion. Among the group of patients with BRCA2-positive BC, a woman was diagnosed at 47 years (2017) with BC and the mutation was identified in 2018; RRSO was performed in 2019 and she was diagnosed in the surgical piece of a serous tubal intraepithelial carcinoma with micro-invasion foci. All four women are healthy and disease-free at present.

Table 1. Pathological findings of RRSO performed in BRCA mutation carriers with and without previous breast cancer.

\begin{tabular}{|c|c|c|c|}
\hline & \multicolumn{3}{|c|}{$\begin{array}{l}\text { RISK-REDUCING SALPINGO-OOPHORECTOMY } \\
\text { ANATOMOPATHOLOGICAL FINDINGS }\end{array}$} \\
\hline & $\begin{array}{c}\text { WITHOUT } \\
\text { PATHOLOGIC } \\
\text { AL FINDINGS }\end{array}$ & BENIGN FINDINGS & MALIGNANT FINDINGS \\
\hline $\begin{array}{c}\text { BRCA1 + } \\
\text { With } \\
\text { Previous } \\
\text { BC } \\
n=23\end{array}$ & $\mathrm{n}=21$ & Ovary: Endometriosis $(\mathrm{n}=1)$ & $\begin{array}{l}\text { Serous Tubal Intraepithelial } \\
\text { Lesion }(n=1)\end{array}$ \\
\hline $\begin{array}{c}\text { BRCA1 + } \\
\text { Without } \\
\text { Previous } \\
\text { BC } \\
\mathrm{n}=19\end{array}$ & $\mathrm{n}=16$ & $\begin{array}{l}\text { Ovary: Serous Cystadenoma } \\
(\mathrm{n}=1)\end{array}$ & $\begin{array}{l}\text { Serous Tubal Carcinoma } \\
(\text { pT1a) }(\mathrm{n}=1) \\
\text { Serous Tubal Intraepithelial } \\
\text { Carcinoma }(\mathrm{n}=1)\end{array}$ \\
\hline $\begin{array}{c}\text { BRCA2 + } \\
\text { With } \\
\text { Previous } \\
\text { BC } \\
\text { n }=29\end{array}$ & $\mathrm{n}=23$ & $\begin{array}{l}\text { Peritubal tissue: Endometriosis } \\
(\mathrm{n}=1) \\
\text { Ovary: Germ Cysts and Teak } \\
\text { Lutein Cysts }(\mathrm{n}=1) \\
\text { Ovary: Epithelial Inclusion Cysts } \\
(\mathrm{n}=2) \\
\text { Ovary: Capillary Hemagioma and } \\
\text { Fibroma }(\mathrm{n}=1)\end{array}$ & $\begin{array}{l}\text { Serous Tubal Intraepithelial } \\
\text { Carcinoma with micro- } \\
\text { invasion foci }(\mathrm{n}=1)\end{array}$ \\
\hline $\begin{array}{c}\text { BRCA2 + } \\
\text { Without } \\
\text { Previous } \\
\text { BC } \\
\mathrm{n}=19\end{array}$ & $\mathrm{n}=15$ & $\begin{array}{l}\text { Ovary: Epithelial Inclusion Cysts } \\
(\mathrm{n}=1) \\
\text { Ovary: Germ Inclusion Cysts } \\
(\mathrm{n}=1) \\
\text { Ovary: Endometriosis }(\mathrm{n}=1) \\
\text { Ovary: Serous Cystadenoma } \\
(\mathrm{n}=1)\end{array}$ & \\
\hline
\end{tabular}


Vol. 5, No. 02; 2021

ISSN: $2581-3366$

In Tables 2 and 3 we show the chronology of events in more detail: age of cancer, genetic diagnosis, and date of RRSO. We also included in Table 2 three patients diagnosed with BC, they were BRCA1 mutation carriers who did not benefit from a RRSO and ended up being diagnosed with high-grade serous carcinoma of the ovary. A fourth patient diagnosed in 2011 with BC at age 40, also a BRCA1 mutation carrier (known since 2017), was diagnosed with serous endometrial carcinoma when a prophylactic hysterectomy with bilateral salpingooophorectomy was performed in 2017.

Table 2. Pathological findings of RRSO performed in BRCA1/2-positive BC patients.

\begin{tabular}{|c|c|c|c|}
\hline \multicolumn{2}{|c|}{ BC Patients (BRCA1+) } & \multicolumn{2}{|c|}{ BC Patients (BRCA2+) } \\
\hline $\begin{array}{l}\text { Bilateral BC } \\
37 \& 37(2004)\end{array}$ & $\begin{array}{l}\text { BRCA1 } \\
\text { 2007:Without pathological } \\
\text { findings }\end{array}$ & $\begin{array}{l}\text { BC } 50(2007) \& \\
\text { Basal cell } \\
\text { carcinoma }\end{array}$ & $\begin{array}{l}\text { BRCA2 (2010) } \\
2011 \text { (HT+ Bilateral SO): Without } \\
\text { pathological findings }\end{array}$ \\
\hline $\begin{array}{l}\text { BC } 63(1991) \& \\
\text { CRC } 79(2008) \& \\
\text { Ovarian cancer } 82 \\
(2011)\end{array}$ & $\begin{array}{l}\text { BRCA1: } 2013 \\
\text { RRSO was not performed } \\
\text { 2011: Ovarian cancer }(82) \text { after } \\
\text { BC and CRC }\end{array}$ & $\begin{array}{l}\text { Bilateral BC } \\
40(2003) \& 47 \\
(2010)\end{array}$ & $\begin{array}{l}\text { BRCA2: } 2012 \\
\text { 2012: Endometriosis in the } \\
\text { peritubal tissue }\end{array}$ \\
\hline $\begin{array}{l}\text { Bilateral BC } \\
28(1990) \& 33 \\
(1996) \\
\end{array}$ & $\begin{array}{l}\text { BRCA1: } 2008 \\
2012 \text { (HT + Bilateral SO): } \\
\text { Without pathological findings }\end{array}$ & BC 44 (2008) & $\begin{array}{l}\text { BRCA2 } \\
\text { 2013: Without pathological findings }\end{array}$ \\
\hline BC 40 (1990) & $\begin{array}{l}\text { BRCA1: } 2013 \\
\text { 2013: Without pathological } \\
\text { findings }\end{array}$ & $\begin{array}{l}\text { Bilateral BC } \\
36(1999) \& 49 \\
(2012)\end{array}$ & $\begin{array}{l}\text { BRCA2: } 2012 \\
\text { 2014: Without pathological findings }\end{array}$ \\
\hline $\begin{array}{l}\text { Bilateral BC } \\
49 \& 49(2012)\end{array}$ & $\begin{array}{l}\text { BRCA1: } 2013 \\
\text { 2014: Without pathological } \\
\text { findings }\end{array}$ & BC 38 (2008) & $\begin{array}{l}\text { BRCA2: } 2014 \\
\text { 2014: Without pathological findings }\end{array}$ \\
\hline $\begin{array}{l}\text { Bilateral BC } \\
36(2003) \& 47 \\
(2014)\end{array}$ & $\begin{array}{l}\text { BRCA1: } 2014 \\
\text { 2015: Without pathological } \\
\text { findings }\end{array}$ & BC 33 (2013) & $\begin{array}{l}\text { BRCA2 } \\
\text { 2014: Without pathological findings }\end{array}$ \\
\hline BC 48 (2013) & $\begin{array}{l}\text { BRCA1: } 2013 \\
\text { 2015: Without pathological } \\
\text { findings }\end{array}$ & $\begin{array}{l}\text { BC } 45(2014) \\
\text { Basal cell } \\
\text { carcinoma }\end{array}$ & $\begin{array}{l}\text { BRCA2: } 2014 \\
\text { 2015: Without pathological findings }\end{array}$ \\
\hline $\begin{array}{l}\text { Bilateral BC } \\
32(1986) \& 55 \\
(2009)\end{array}$ & $\begin{array}{l}\text { BRCA1: } 2015 \\
\text { 2015: Without pathological } \\
\text { findings }\end{array}$ & BC 27 (1999) & $\begin{array}{l}\text { BRCA2: } 2014 \\
\text { 2015: Without pathological findings }\end{array}$ \\
\hline BC 45 (2012) & $\begin{array}{ll}\text { BRCA1: } 2014 & \\
\text { 2015: Without pathological } \\
\text { findings }\end{array}$ & BC 27 (1999) & $\begin{array}{l}\text { BRCA2: } 2014 \\
\text { 2015: Without pathological findings }\end{array}$ \\
\hline $\begin{array}{l}\text { BC } 45(1998) \& \\
\text { Ovarian cancer } 60 \\
(2015)\end{array}$ & $\begin{array}{l}\text { BRCA } 1: 2016 \\
\text { RRSO was not performed } \\
\text { 2015: Ovarian cancer }(60) \\
\end{array}$ & BC $56(2010)$ & $\begin{array}{l}\text { BRCA2: } 2014 \\
\text { 2015: Without pathological findings }\end{array}$ \\
\hline BC 35 (2016) & $\begin{array}{l}\text { BRCA1: } 2016 \\
\text { 2016: Without pathological } \\
\text { findings }\end{array}$ & BC 34 (2004) & $\begin{array}{l}\text { BRCA2:2011 } \\
\text { 2015: Ovarian Germ Cysts, Teak } \\
\text { Lutein Cysts }\end{array}$ \\
\hline $\begin{array}{l}\text { Bilateral BC } \\
45(1997) \& 63 \\
(2015) \\
\end{array}$ & $\begin{array}{l}\text { BRCA1: } 2015 \\
\text { 2016: Without pathological } \\
\text { findings }\end{array}$ & BC 59 (2013) & $\begin{array}{l}\text { BRCA2: } 2014 \\
\text { 2015: Without pathological findings }\end{array}$ \\
\hline BC 43 (2011) & $\begin{array}{l}\text { BRCA1: } 2016 \\
\text { 2016: Without pathological } \\
\text { findings }\end{array}$ & BC 44 (2016) & $\begin{array}{l}\text { BRCA2 (2016) } \\
\text { 2016: Without pathological findings }\end{array}$ \\
\hline
\end{tabular}


Vol. 5, No. 02; 2021

ISSN: 2581-3366

\begin{tabular}{|c|c|c|c|}
\hline BC 27 (2015) & $\begin{array}{l}\text { BRCA1 } \\
\text { 2017: Without pathological } \\
\text { findings }\end{array}$ & $\begin{array}{l}\text { Bilateral BC } \\
39 \quad(2008) \& \quad 44 \\
(2014)\end{array}$ & $\begin{array}{l}\text { BRCA2:2020 } \\
\text { 2016:Ovary with Epithelial } \\
\text { Inclusion Cysts }\end{array}$ \\
\hline $\begin{array}{l}\text { Bilateral BC } \\
42 \quad(2000) \quad \& 53 \\
(2011)\end{array}$ & $\begin{array}{l}\text { BRCA1 } \\
\text { 2017: Without pathological } \\
\text { findings }\end{array}$ & BC 53 (2013) & $\begin{array}{l}\text { BRCA2: } 2015 \\
\text { 2016:Ovary with Epithelial } \\
\text { Inclusion Cysts }\end{array}$ \\
\hline $\begin{array}{l}\text { Bilateral BC } \\
58 \quad(2008) \quad \& \quad 62 \\
(2012)\end{array}$ & $\begin{array}{l}\text { BRCA1: } 2017 \\
\text { 2017: Without pathological } \\
\text { findings }\end{array}$ & BC 44 (1991) & $\begin{array}{l}\text { BRCA2: } 2013 \\
\text { 2016: Without pathological findings } \\
\text { Later: Pancreatic Cancer }\end{array}$ \\
\hline BC 40 (2001) & $\begin{array}{l}\text { BRCA1: } 2016 \\
\text { 2017: Without pathological } \\
\text { findings }\end{array}$ & BC 52 (2002) & $\begin{array}{l}\text { BRCA2: } 2011 \\
\text { 2016: Without pathological findings }\end{array}$ \\
\hline BC 47 (2005) & $\begin{array}{l}\text { BRCA1:2014 } \\
\text { 2017: Without pathological } \\
\text { findings }\end{array}$ & $\begin{array}{l}\text { Bilateral BC } \\
40 \quad(1995) \quad \& 47 \\
(2002)\end{array}$ & $\begin{array}{l}\text { BRCA2: } 2015 \\
\text { 2017: Without pathological findings }\end{array}$ \\
\hline BC 46 (2011) & $\begin{array}{l}\text { BRCA1:2016 } \\
\text { 2017: Serous Tubal } \\
\text { Intraepithelial Lesion }\end{array}$ & $\begin{array}{l}\text { Bilateral BC } \\
42(2006) \& 53 \\
(2017)\end{array}$ & $\begin{array}{l}\text { BRCA2: } 2012 \\
\text { 2017: Without pathological findings }\end{array}$ \\
\hline BC 33 (2011) & $\begin{array}{l}\text { BRCA1:2011 } \\
\text { 2017: Without pathological } \\
\text { findings }\end{array}$ & BC $42(2011)$ & $\begin{array}{l}\text { BRCA2: } 2016 \\
\text { 2017: Without pathological findings }\end{array}$ \\
\hline BC 40 (2017) & $\begin{array}{l}\text { BRCA } 1: 2017 \\
\text { 2017: Ovary with } \\
\text { Endometriotic Cysts }\end{array}$ & BC 36 (1998) & $\begin{array}{l}\text { BRCA2: } 2016 \\
\text { 2017: Without pathological findings }\end{array}$ \\
\hline $\begin{array}{l}\text { BC } 40(2011) \& \\
\text { Endometrial cancer } \\
46(2017)\end{array}$ & $\begin{array}{l}\text { BRCA1: } 2017 \\
\text { 2017 (HT + Bilateral SO): } \\
\text { Endometrial Serous } \\
\text { Carcinoma }\end{array}$ & $\begin{array}{l}\text { Bilateral BC } \\
43 \quad(1989) \& \quad 65 \\
(2011)\end{array}$ & $\begin{array}{l}\text { BRCA2: } 2017 \\
\text { 2018: Ovarian capillary } \\
\text { hemangioma and Ovarian fibroma }\end{array}$ \\
\hline BC 37 (1987) & $\begin{array}{l}\text { BRCA1: } 2019 \\
\text { 2017: v }\end{array}$ & BC 47 (2017) & $\begin{array}{l}\text { BRCA2: } 2018 \\
\text { 2019: Without pathological findings }\end{array}$ \\
\hline BC 49 (2012) & $\begin{array}{l}\text { BRCA1: } 2018 \\
\text { 2018: Without pathological } \\
\text { findings }\end{array}$ & BC 47 (2017) & $\begin{array}{l}\text { BRCA2: } 2018 \\
\text { 2019:Serous Tubal Intraepithelial } \\
\text { Carcinoma with micro-invasion } \\
\text { foci }\end{array}$ \\
\hline $\begin{array}{l}\text { BC } 33(2003) \& \\
\text { Ovarian cancer } 49 \\
(2018)\end{array}$ & $\begin{array}{l}\text { BRCA1: } 2019 \\
\text { RRSO was not performed } \\
\text { 2018: Ovarian cancer (49), } \\
\text { after BC }\end{array}$ & $\begin{array}{l}\text { BC } 62(2005) \& \\
\text { CRC \& Papillary } \\
\text { Thyroid Carcinoma }\end{array}$ & $\begin{array}{l}\text { BRCA2: } 2017 \\
\text { 2019: Without pathological findings }\end{array}$ \\
\hline BC 33 (2012) & $\begin{array}{l}\text { BRCA1: } 2013 \\
\text { 2020: Without pathological } \\
\text { findings }\end{array}$ & $\begin{array}{l}\text { BC } 64(2018) \& \\
\text { Basal cell } \\
\text { carcinoma }\end{array}$ & $\begin{array}{l}\text { BRCA2:2018 } \\
\text { 2019: Without pathological findings }\end{array}$ \\
\hline BC 29 (2007) & $\begin{array}{l}\text { BRCA1: } 2019 \\
\text { 2020: Without pathological } \\
\text { findings }\end{array}$ & BC 35 (2017) & $\begin{array}{l}\text { BRCA2: } 2017 \\
\text { 2018: Criopreservación de ovocitos } \\
\text { 2019: Without pathological findings }\end{array}$ \\
\hline & & BC 30 (2004) & $\begin{array}{l}\text { BRCA2: } 2014 \\
\text { 2019: Without pathological findings }\end{array}$ \\
\hline & & $\begin{array}{l}\text { Bilateral BC } \\
41 \quad(2003) \quad \& \quad 57 \\
(2019)\end{array}$ & $\begin{array}{l}\text { BRCA2: } 2019 \\
\text { 2020: Without pathological findings }\end{array}$ \\
\hline
\end{tabular}

Notes: CRC: colorectal carcinoma; HT: Hysterectomy; SO: salpingo-oophorectomy 


\section{International Journal of Medical Science and Health Research}

Vol. 5, No. 02; 2021

ISSN: $2581-3366$

Table 3. Pathological findings of RRSO performed in healthy BRCA1/2 mutation carriers.

\begin{tabular}{|c|c|c|c|}
\hline \multicolumn{2}{|c|}{ BRCA1-Positive Women (without BC) } & \multicolumn{2}{|c|}{ BRCA2-Positive Women (without BC) } \\
\hline BRCA1:2006 & $\begin{array}{l}\text { 2016:Serous Tubal } \\
\text { Carcinoma(pT1a) }\end{array}$ & $\begin{array}{l}\text { BRCA2: } \\
2010\end{array}$ & $\begin{array}{l}\text { 2016: Ovary with Epithelial } \\
\text { Inclusion Cysts }\end{array}$ \\
\hline $\begin{array}{l}\text { BRCA1: } \\
2009\end{array}$ & 2010: Without pathological findings & $\begin{array}{l}\text { BRCA2: } \\
2010\end{array}$ & $\begin{array}{l}\text { 2014: Without pathological findings } \\
\text { 2015: Thymoma }\end{array}$ \\
\hline $\begin{array}{l}\text { BRCA1: } \\
2009\end{array}$ & 2011: Without pathological findings & $\begin{array}{l}\text { BRCA2: } \\
2011\end{array}$ & 2016: Without pathological findings \\
\hline $\begin{array}{l}\text { BRCA1: } \\
2009\end{array}$ & 2011: Without pathological findings & $\begin{array}{l}\text { BRCA2: } \\
2014\end{array}$ & 2014: Without pathological findings \\
\hline $\begin{array}{l}\text { BRCA1: } \\
2011\end{array}$ & 2013: Without pathological findings & $\begin{array}{l}\text { BRCA2: } \\
2014\end{array}$ & 2014: Without pathological findings \\
\hline $\begin{array}{l}\text { BRCA1: } \\
2011\end{array}$ & $\begin{array}{l}\text { 2014: Without pathological findings } \\
\text { 2020:Serous Peritoneum } \\
\text { Carcinoma }\end{array}$ & $\begin{array}{l}\text { BRCA2: } \\
2014\end{array}$ & 2014: Without pathological findings \\
\hline $\begin{array}{l}\text { BRCA1: } \\
2011\end{array}$ & $\begin{array}{l}2016(\mathrm{HT}+\text { Bilateral SO)Without } \\
\text { pathological findings } \\
\text { 2018: sarcomatoid carcinoma of the } \\
\text { bladder }\end{array}$ & $\begin{array}{l}\text { BRCA2: } \\
2014\end{array}$ & 2014: Withouth pathological findings \\
\hline $\begin{array}{l}\text { BRCA1: } \\
2013\end{array}$ & $\begin{array}{l}\text { 2014: Without pathological findings } \\
\text { 2019: Lung adenocarcinoma }\end{array}$ & $\begin{array}{l}\text { BRCA2: } \\
2014\end{array}$ & 2014: Withouth pathological findings \\
\hline $\begin{array}{l}\text { BRCA1: } \\
2013\end{array}$ & 2014: Without pathological findings & $\begin{array}{l}\text { BRCA2: } \\
2014\end{array}$ & $\begin{array}{l}\text { 2015: Serous Cystadenoma of the } \\
\text { Ovary }\end{array}$ \\
\hline $\begin{array}{l}\text { BRCA1: } \\
2014\end{array}$ & 2016: Without pathological findings & $\begin{array}{l}\text { BRCA2: } \\
2014\end{array}$ & $\begin{array}{l}\text { 2014: Without pathological findings } \\
\text { 2015: In Situ BC }\end{array}$ \\
\hline $\begin{array}{l}\text { BRCA1: } \\
2015\end{array}$ & 2016: Without pathological findings & $\begin{array}{l}\text { BRCA2: } \\
2014\end{array}$ & $\begin{array}{l}\text { 2014: Without pathological findings } \\
\text { 2016: Infiltrating BC }\end{array}$ \\
\hline $\begin{array}{l}\text { BRCA1: } \\
2015\end{array}$ & $\begin{array}{l}\text { 2019: Prophylactic Mastectomy } \\
\text { 2019: Serous Tubal Intraepithelial } \\
\text { Carcinoam }\end{array}$ & $\begin{array}{l}\text { BRCA2: } \\
2014\end{array}$ & $\begin{array}{l}\text { 2014: Without pathological findings } \\
\text { 2016: Infiltrating BC }\end{array}$ \\
\hline $\begin{array}{l}\text { BRCA1: } \\
2017\end{array}$ & 2018: Without pathological findings & $\begin{array}{l}\text { BRCA2: } \\
2014\end{array}$ & $\begin{array}{l}\text { 2015: Without pathological findings } \\
\text { Previous: Melanoma (1998) }\end{array}$ \\
\hline $\begin{array}{l}\text { BRCA1: } \\
2018\end{array}$ & 2020: Without pathological findings & $\begin{array}{l}\text { BRCA2: } \\
2014\end{array}$ & $\begin{array}{l}\text { 2016: Ovary with Germ Inclusion } \\
\text { Cysts }\end{array}$ \\
\hline $\begin{array}{l}\text { BRCA1: } \\
2019\end{array}$ & 2020: Without pathological findings & $\begin{array}{l}\text { BRCA2: } \\
2015\end{array}$ & 2015: Without pathological findings \\
\hline $\begin{array}{l}\text { BRCA1: } \\
2019\end{array}$ & 2020: Without pathological findings & $\begin{array}{l}\text { BRCA2: } \\
2015\end{array}$ & $\begin{array}{l}\text { 2016: Without pathological findings } \\
\text { 2018: Infiltrating BC }\end{array}$ \\
\hline BRCA1 & $\begin{array}{l}2016(\mathrm{HT}+\text { Bilateral SO): Without } \\
\text { pathological findings }\end{array}$ & $\begin{array}{l}\text { BRCA2: } \\
2017\end{array}$ & 2018: Ovarian Endometriosis \\
\hline BRCA1 & 2018:Ovary: Serous Cystadenoma & $\begin{array}{l}\text { BRCA2: } \\
2019\end{array}$ & 2020: Without pathological findings \\
\hline $\begin{array}{l}\text { BRCA1: } \\
2017\end{array}$ & $\begin{array}{l}\text { 2020: Without pathological findings } \\
\text { 2020: Infiltrating } \mathrm{BC}(38)\end{array}$ & $\begin{array}{l}\text { BRCA2: } \\
2019\end{array}$ & 2020: Without pathological findings \\
\hline
\end{tabular}

Notes: HT: Hysterectomy; SO: salpingo-oophorectomy. 
Vol. 5, No. 02; 2021

ISSN: 2581-3366

One healthy BRCA1 mutation carrier (known since 2011) underwent a RRSO in 2014 without pathological findings, and presented six years later (2020) with a serous peritoneal carcinoma. Serous tubal carcinomas were found in two of the $19(10.5 \%)$ healthy BRCA1 mutation carriers who underwent RRSO: one infiltrating serous tubal carcinoma (pT1a) and another serous tubal intraepithelial carcinoma. No malignant neoplasms were found in RRSO performed in healthy BRCA2 mutation carriers.

\section{Discussion}

Ovarian cancer is a heterogeneous disease. More than $90 \%$ of ovarian cancers are epithelial: they can arise from the epithelium of the ovarian surface, but also from the fallopian tubes, foci of endometriosis, or in the peritoneum [1]. Four main types are distinguished within epithelial ovarian cancer: serous, endometrioid, mucinous, and clear cell. Each of them shows gene expression patterns that correlate with their morphological counterparts in normal tissues: serous tumors were correlated with the fallopian tubes, mucinous tumors with the colonic mucosa, and endometrioid and clear cells with the endometrium [2].

Theories of carcinogenesis indicate that serous ovarian cancers derive from the epithelium of the fallopian tube and affect the ovary secondarily, highlighting two hypotheses for their pathogenesis. According to the first, the precursors of ovarian cancer develop in the fimbriae from an occult serous tubal intraepithelial carcinoma (STIC -serous tubal intraepithelial carcinoma-), subsequently involving the ovary. The second theory supports the implantation of normal fimbrial epithelium on the ovarian surface during ovulation, resulting in a cortical inclusion cyst where malignant transformation can arise [3, 4].

Between $15-20 \%$ of patients with ovarian cancer are BRCA1/2 mutation carriers. Although a family history of breast and ovarian cancer is strongly correlated with the detection of a BRCA1/2 mutation, up to $50 \%$ of women with BRCA-positive ovarian cancer have no family history of cancer. This supports the importance and indication of genetic testing for all women with a personal diagnosis of epithelial ovarian cancer (including fallopian and peritoneal cancer), regardless of age at diagnosis and family history [5]. Carriers of these mutations are more likely to be diagnosed with high-grade serous ovarian cancer than other histologic subtypes.

Therefore, the increased risk of ovarian and fallopian tube cancer in BRCA1/2 positive women is well documented. For this reason and since there is no effective screening for this type of cancer, RRSO is the surgery of choice in women carrying BRCA mutation who have completed their birth desire.

RRSO is recommended from 35-40 years for BRCA1-positive and from 40-45 years for BRCA2-positive women, although the age of the youngest affected person in the family should always be considered [6]. This age difference is established because BRCA1 mutation carriers tend to develop ovarian cancer at younger ages [7]. 
Vol. 5, No. 02; 2021

ISSN: 2581-3366

RRSO has been shown to decrease the risk of primary ovarian, tubal, and peritoneal cancer by $80 \%$ in BRCA $1 / 2$ carriers [8]. A residual risk of $1-4.3 \%$ of primary peritoneal carcinoma has been reported $[8,9]$. In our series, we have also been able to confirm that $1.1 \%$ of women with a previous RRSO ( 1 of 90) without presenting relevant pathological findings, were diagnosed six years later with peritoneal serous carcinoma. RRSO is estimated to confer a $77 \%$ reduction in allcause mortality [10].

Regarding the reduction in the risk of $\mathrm{BC}$ associated with RRSO, the current data are controversial: initial studies showed a risk reduction in BRCA1/2 mutation carriers of $56 \%$ and $43 \%$ respectively [11], several studies corroborate this risk reduction of $51 \%$ [8], but more recent ones did not validate this protective effect $[12,13]$. A study of the year 2020 showed that RRSO in premenopause decreased the risk of BC in BRCA1 but not in BRCA2 mutation carriers [14].

Occult ovarian and fallopian cancers removed prophylactically have been reported in women carrying a BRCA mutation, ranging from 2-12\% [15-18]. For this reason, a standardized surgical protocol is important as well as a detailed and protocolzed histological study of the surgical pieces. In our study, in four of the 90 RRSOs performed (4.4\%), malignant lesions were found at the level of the fallopian tube (serous carcinomas): one of the cases was infiltrating (pT1a), another was intraepithelial with foci of micro-invasion and in the remaining two, the lesion was exclusively intraepithelial.

RRSO has a specific surgical protocol, that we must follow [19]: minimally invasive laparoscopic approach, survey of the abdominopelvic cavity (upper abdomen, bowel surfaces, omentum, appendix, and pelvic organs), biopsy of any abnormal peritoneal finding, pelvic washing for cytology (50 cc of normal saline instilled and aspirated immediately), bilateral salpingo-oophorectomy (including: $2 \mathrm{~cm}$ of infundibulopelvic ligament, all of the fallopian tubes from the uterine horn and especially the peritoneum surrounding the ovaries and fallopian tubes, as well as areas of underlying adhesions between the tube/ovary, and pelvic wall), minimize fallopian tube/ovarian manipulation to avoid traumatic exfoliation, place the ovaries and tubes in an endo bag for retrieval from the pelvis. Interval salpingectomy and deferral of oophorectomy are not indicated.

Serial sectioning and careful microscopic examination of the bilateral salpingo-oophorectomy specimen is a well-established practice. This is done using the protocol SEE-FIM (Sectioning and Extensively Examining the Fimbriated End), which involves pathological revision of the entire sample in 2 to $3 \mathrm{~mm}$ sections, as well as the longitudinal section of the fimbria [20,21] (Figure 1). The main predictor for detecting hidden malignancy in women at high risk for ovarian cancer who are going to undergo RRSO is to adhere to the surgical-pathological protocol. It is important because following a standardized protocol is associated with higher rates of diagnosis. 
Vol. 5, No. 02; 2021

ISSN: $2581-3366$

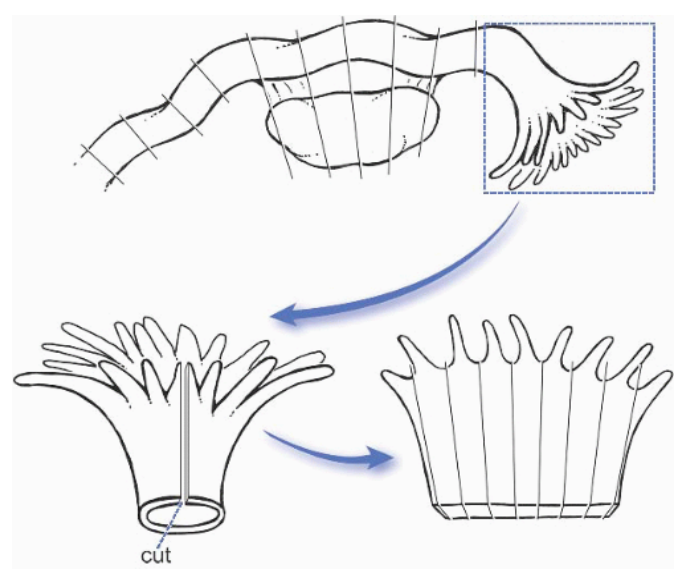

Figure 1. Serial section of the fallopian tube and ovary, from a sample of RRSO (SEE-FIM protocol). The fimbriated end should be amputated from the rest of the tube and sectioned serially at $2 \mathrm{~mm}$ intervals along the long axis. The remainder of the fallopian tube as well as the ovary should be cut perpendicular to the long axis at $2 \mathrm{~mm}$ intervals.

For the non-BRCA genes (BRCAX), various studies show that carriers of BRIP1, RAD51C, and RAD51D have a moderate risk of ovarian cancer, so RRSO is recommended; although there is no evidence of at what age it is recommended to perform RRSO (the guidelines recommend its consideration in peri/menopause: $45-50$ years) [6]. The cumulative risk of ovarian cancer for Lynch syndrome carriers is $60 \%$, therefore RRSO is also recommended in these patients. In general, risk-reducing surgery (hysterectomy + bilateral salpingo-oophorectomy) is recommended in women with Lynch syndrome at similar ages to BRCA2 carriers (around 40-45 years) $[22,23]$, although guidelines such as those of the National Comprehensive Cancer Network (NCCN) do not specify age [24] because there is variability according to the type of mutation and family history [25]. For the PALB2, ATM, CHEK2, or NBN genes, it is recommended to consider RRSO based on family history.

The benefits and side effects associated with RRSO and subsequent surgical menopause need to be weighed. In premenopausal women, oophorectomy increases the risk of cardiovascular morbidity, osteoporosis, and associated endocrine symptoms including hot flashes and impaired sexual function. Fear of these symptoms can influence the decision to undergo this procedure. In general, for BRCA1/2 mutation carriers, the impact on the quality of life-related to surgical menopause is outweighed by the reduction in cancer risk [26, 27]. It is recommended that a gynecologist-oncologist explain the risks/benefits and help patients who are considering RRSO to understand how it can affect the quality of life and assess treatment options, if necessary. The role of hormone replacement therapy in BRCA1/2 mutation carriers submitted to RRSO has been controversial. Although the evidence is limited, hormone therapy has a number of reported benefits and does not appear to affect BC risk [28]. 
Vol. 5, No. 02; 2021

ISSN: $2581-3366$

\section{Conclusion}

In our series of 90 RRSOs performed on women carrying BRCA1/2 mutations, with and without previous breast cancer, 4 women $(4.4 \%)$ were diagnosed with malignant lesions in the fallopian epithelium (all of them in early stages).In the absence of new imaging techniques or new serum markers to predictably identify early-stage ovarian and fallopian cancer, RRSO remains the best option for women at high risk of developing ovarian and fallopian cancer. The main predictor for detecting hidden malignancy in women at high risk for ovarian cancer who are going to undergo RRSO is to adhere to the surgical-pathological protocol. However, we must always bear in mind that there will always be a residual risk of primary peritoneal carcinoma after RRSO $(1.1 \%$ in our series).

We emphasize the importance of performing a germline testing for all women diagnosed with epithelial ovarian cancer (including fallopian and peritoneal cancer), regardless of age at diagnosis (NCCN Guidelines). We also recall the importance of trying to identify all women with hereditary breast and ovarian cancer syndrome (BRCA1/2 mutation carriers) and those carriers of other mutations with increased risk of ovarian cancer.

Our data support the indication for RRSO in selected high-risk patients; and recall the importance of the identification of high-risk patients, in order to offer genetic counseling and preventive measures, both for patients and their families.

\section{Authors' Citation:}

Rodríguez-Fernández V, Cameselle-Cortizo L, González L, Figueiredo-Alonso E, Lamas MJ, Valdés-Pons J, Cameselle-Teijeiro JF.

\section{Conflicts of Interest}

The authors declare that there is no conflict of interest regarding the publication of this paper.

\section{Funding}

All authors have no source of funding.

\section{Acknowledgments}

We thank our colleagues from the Breast Unit of Meixoeiro Hospital and Gynecological Service of Álvaro Cunqueiro and Povisa Hospital who provided insight, data and expertise that greatly assisted the research and publication of this article.

\section{References}

1.- Bast RC Jr, Hennessy B, Mills GB. The biology of ovarian cancer: new opportunities for translation. NatRev Cancer 2009; 9 (6):415-28. doi: 10.1038/nrc2644.

2.- Marquez RT, Baggerly KA, Patterson AP, Liu J, Broaddus R, Frumovitz M et al. Patterns of gene expression in different histotypes of epithelial ovarian cancer correlate with those 
Vol. 5, No. 02; 2021

ISSN: 2581-3366

in normal fallopian tube, endometrium, and colon. Clin Cancer Res 2005;11(17):611626. doi: 10.1158/1078-0432.CCR-04-2509.

3.- Kurman RJ. Origin and molecular pathogenesis of ovarian high-grade serous carcinoma. Ann Oncol 2013;24 Suppl 10:x16-21. doi: 10.1093/annonc/mdt463

4.- Sama AR, Schilder RJ. Refractoryfallopiantube carcinoma-current perspectives in pathogenesis and management. Int J WomensHealth. 2014 Jan 28; 6:149-57. doi: 10.2147/IJWH.S40889.

5.- Daly MB, Pal T, Berry MP, Buys SS, Dickson P, Domchek SM et al. Genetic / Familial High-RiskAssessment: Breast, Ovarian, and Pancreatic, Version 2.2021, NCCN ClinicalPracticeGuidelines in Oncology. J NatlComprCancNetw. 2021; 19 (1): 77-102. doi: 10.6004 / jnccn.2021.0001.

6.- González-Santiago S, Ramón Y Cajal T, Aguirre E, Alés-Martínez JE, Andrés R, Balmaña J et al. SEOM clinical guidelines in hereditary breast and ovarian cancer (2019). Clin Transl Oncol. 2020; 22 (2): 193-200. doi: 10.1007 / s12094-019-02262-0.

7.- Finch AP, Lubinski J, Møller P, Singer CF, Karlan B, Senter L et al. Impact of oophorectomy on cancer incidence and mortality in women with a BRCA1 or BRCA2 mutation. J Clin Oncol. 2014 May 20; 32 (15): 1547-53. doi: 10.1200 / JCO.2013.53.2820.

8.- Rebbeck TR, Kauff ND, Domchek SM. Meta-analysis of risk reduction estimates associated with risk-reducing salpingo-oophorectomy in BRCA1 or BRCA2 mutation carriers. J Natl Cancer Inst 2009; 101 (2): 80-7.

9.- Kauff ND, Domchek SM, Friebel TM, Robson ME, Lee J, Garber JE et al. Risk-reducing salpingo-oophorectomy for the prevention of BRCA1- and BRCA2-associated breast and gynecologic cancer: a multicenter, prospective study. J Clin Oncol 2008; 26 (8): 1331-7. doi: 10.1200 / JCO.2007.13.9626.

10.- Xiao YL, Wang K, Liu Q, Li J, Zhang X, Li HY. Risk Reduction and Survival Benefit of Risk-Reducing Salpingo-oophorectomy in Hereditary Breast Cancer: Meta-analysis and Systematic Review. Clin Breast Cancer. 2019; 19 (1): e48-e65. doi: 10.1016 / j.clbc.2018.09.011.].

11.- Eisen A, Lubinski J, Klijn J, Moller P, Lynch HT, Offit K et al. Breast cancer risk following bilateral oophorectomy in BRCA1 and BRCA2 mutation carriers: an international casecontrol study. J Clin Oncol 2005; 23 (30): 7491-6. doi: 10.1200 / JCO.2004.00.7138.

12.- Heemskerk-Gerritsen BA, Seynaeve C, van Asperen CJ, Ausems MG, Collée JM, van Doorn $\mathrm{HC}$ et al. Breast cancer risk after salpingo-oophorectomy in healthy BRCA1/2 mutation carriers: revisiting the evidence for risk reduction. J Natl Cancer Inst 2015; 107 (5): djv033. doi: 10.1093 / jnci / djv033. 
Vol. 5, No. 02; 2021

ISSN: 2581-3366

13.- Kotsopoulos J, Huzarski T, Gronwald J, Singer CF, Moller P, Lynch HT et al. Bilateral Oophorectomy and Breast Cancer Risk in BRCA1 and BRCA2 Mutation Carriers. J Natl Cancer Inst 2016; 109 (1): djw177. doi: 10.1093 / jnci / djw177.

14.- Stjepanovic N, Villacampa G, Nead KT, Torres-Esquius S, Melis GG, Nathanson KL et al. Association of premenopausal risk-reducing salpingo-oophorectomy with breast cancer risk in BRCA1 / 2 mutation carriers: Maximizing bias-reduction. Eur J Cancer 2020; 132: 53-60. doi: 10.1016 / j.ejca.2020.03.009.

15.- Powell CB, Chen LM, McLennan J, Crawford B, Zaloudek C, Rabban JT et al. Riskreducing salpingo-oophorectomy (RRSO) in BRCA mutation carriers: experience with a consecutive series of 111 patients using a standard zedsurgical-pathological protocol. Int J GynecolCancer 2011; 21 (5):846-51. doi: 10.1097/IGC.0b013e31821bc7e3.

16.- Rhiem K, Foth D, Wappenschmidt B, Gevensleben H, Büttner R, Ulrich U, Schmutzler RK. Risk-reducingsalpingo-oophorectomy in BRCA1 and BRCA2 mutationcarriers. Arch Gynecol Obstet 2011;283 (3):623-7. doi: 10.1007/s00404-010-1476-3.

17.- Mingels MJ, Roelofsen T, van der Laak JA, de Hullu JA, van Ham MA, Massuger LF, Bulten J, Bol M. Tubal epitelial lesions in salpingo-oophorectomy specimens of BRCA-mutation carriers and controls. Gynecol Oncol. 2012;127(1):88-93. doi: 10.1016/j.ygyno.2012.06.015.

18.- Sherman ME, Piedmonte M, Mai PL, Ioffe OB, Ronnett BM, Van Le L, Ivanov I, Bell MC, Blank SV, DiSilvestro P, Hamilton CA, Tewari KS, Wakeley K, Kauff ND, Yamada SD, Rodriguez G, Skates SJ, Alberts DS, Walker JL, Minasian L, Lu K, Greene MH. Pathologic findings at risk-reducing salpingo-oophorectomy: primary results from Gynecologic Oncology Group Trial GOG-0199. J Clin Oncol 2014;32 (29):3275-83. doi: 10.1200/JCO.2013.54.1987.

19.- National Comprehensive Cancer Network (NCCN). NCCN Guidelines: Ovarian Cancer (Version 2.2020). Avaliable at: https://www.nccn.org/professionals/physician gls/pdf/ovarian.pdf.

20.- Medeiros F, Muto MG, Lee Y, Elvin JA, Callahan MJ, Feltmate C, Garber JE, Cramer DW, Crum CP. The tubal fimbria is a preferred site for early adenocarcinoma in women with family ovarian cancer syndrome. Am J Surg Pathol. 2006; 30 (2): 230-6. doi: 10.1097 / 01.pas.0000180854.28831.77.

21.- College of American Pathologists (CAP). Protocol for the examination of specimens with $\begin{array}{llll}\text { carcinoma of the } & \text { ovary. } & \end{array}$ https:/www.cap.org/apps/docs/committees/cancer/cancer_protocols/2009/Ovary_09pro tocol.pdf 
Vol. 5, No. 02; 2021

ISSN: 2581-3366

22.- ACOG Practice Bulletin No. 147: Lynch syndrome. Obstet Gynecol. 2014 Nov;124(5):1042-1054. doi: 10.1097/01.AOG.0000456325.50739.72.

23.- Syngal S, Brand RE, Church JM, Giardiello FM, Hampel HL, Burt RW; American College of Gastroenterology. ACG clinical guideline: Genetic testing and management of hereditary gastrointestinal cancer syndromes. Am J Gastroenterol 2015;110(2):223-62; quiz 263. doi: 10.1038/ajg.2014.435.

24.- National Comprehensive Cancer Network (NCCN). NCCN Guidelines: Colon Cancer. https://www.nccn.org/professionals/physician_gls/pdf/genetics_colon.pdf.

25.- Dominguez-Valentin M, Crosbie EJ, Engel C, Aretz S, Macrae F, Winship I et al. Riskreducing hysterectomy and bilateral salpingo-oophorectomy in female heterozygotes of pathogenic mismatch repair variants: a Prospective Lynch Syndrome Database report. GenetMed 2020. doi: 10.1038/s41436-020-01029-1

26.- Pezaro C, James P, McKinley J, Shanahan M, Young MA, Mitchell G. The consequences of risk-reducing salpingo-oophorectomy: the case for a coordinated approach to long-term follow-up postsurgical menopause. Fam Cancer 2012;11(3):403-10. doi: 10.1007/s10689-012-9527-5.

27.-Hooker GW, King L, Vanhusen L, Graves K, Peshkin BN, Isaacs C et al. Long-term satisfaction and quality of life following risk reducing surgery in BRCA1/2 mutation carriers. Hered Cancer Clin Pract 2014;12(1):9. doi: 10.1186/1897-4287-12-9.

28.- Gordhandas S, Norquist BM, Pennington KP, Yung RL, Laya MB, Swisher EM. Hormone replacement therapy after risk-reducing salpingo-oophorectomy in patients with BRCA1 or BRCA2 mutations; a systematic review of risks and benefits. Gynecol Oncol 2019; 153(1): 192-200. doi: 10.1016/j.ygyno.2018.12.014. 